\section{Development of melanocortin circuits}

The melanocortin system is known to be involved in energy homeostasis; however, the mechanisms underlying the development of melanocortin circuits (and therefore their role in metabolic disorders such as obesity) have been largely unknown. New research now suggests that class 3 semaphorin signalling is a key factor in the development of melanocortin circuits in the hypothalamus, and that genetic variants that disrupt this pathway are associated with obesity in humans.

Previous work has shown that semaphorins are axon guidance molecules that direct the development of neuronal circuits. "Based on these studies, we hypothesized that semaphorin 3 signalling might be required for the normal development of pro-opiomelanocortin (POMC) circuits and that the loss of signalling in POMC neurons would have an effect on weight and energy balance," explain authors Sebastien Bouret and Sadaf Farooqi.

Analysis of exome sequencing data from 573 patients with severe early-onset obesity allowed the researchers to identify
40 variants in the genes encoding the class 3 semaphorins and their receptors. These rare variants were enriched in patients with severe early-onset obesity compared with control individuals. In mice, deletion of a semaphorin 3 receptor disrupted the development of POMC neurons in the hypothalamus and resulted in reduced energy expenditure and weight gain.

"This study demonstrates for the first time that axon guidance cues are involved in the development of brain circuits involved in energy balance," say Bouret and Farooqi. studies to characterize the effect of semaphorins in mature hypothalamic neurons, and to investigate the role of other axon guidance molecules. "These further studies will inform understanding of the mechanisms that underlie human disorders characterized by hypothalamic dysfunction," conclude Bouret and Farooqi.

Claire Greenhill

ORIGINAL ARTICLE van der Klaauw, A. A. et al. Human semaphorin 3 variants link melanocortin circuit development and energy balance. Cell https://doi.org/10.1016/j.cell. 2018.12.009 (2019) The researchers are planning additional

\title{
An insulin pill
}

Patients with type 2 diabetes mellitus (T2DM) currently administer insulin via a subcutaneous injection. Oral insulin therapy, however, holds multiple potential benefits; for example, it would be convenient for patients and would reduce peripheral hyperinsulinaemia.

While oral insulin preparations have been explored for many years in both animal models and patients, a promising formulation for clinical application has not yet been created. 1338, a long-acting oral insulin preparation that was the subject of a recent phase II clinical trial, could be an important stepping stone on this path. "Although the present formulation will not be taken forward, we have shown that insulin can be effectively administered orally to patients with T2DM," says Inge Halberg, lead author of the study.

In the double-blind, randomized controlled trial, 49 patients with T2DM who were currently taking metformin or another oral anti-diabetic drug were given either IGlar, a subcutaneously administered insulin preparation, or 1338 tablets once daily in the morning for 8 weeks. At the end of the study, the authors assessed plasma glucose concentration in both groups and found that both treatments were equally efficacious at lowering blood glucose values measured at ten time points throughout the day.

Despite these encouraging findings, the authors note that total and within-patient variability of fasting blood-glucose levels were higher in the 1338 group than in the IGlar group.

"The potential benefits of oral basal insulin therapy versus other treatment options still need to be investigated in adequately powered, long-term clinical trials in various patient populations with diabetes mellitus," explains Halberg. "The findings of the present study, however, are likely to encourage the further development of oral insulin products."

Anna Kriebs, Associate Editor, Nature Communications

ORIGINAL ARTICLE Halberg, I. B. et al. Efficacy and safety of oral basal insulin versus subcutaneous insulin glargine in type 2 diabetes: a randomised, double-blind, phase 2 trial. Lancet Diabetes Endocrinol. https://doi.org/10.1016/S2213-8587 (18)30372-3 (2019)

\section{A new metabolic role for TGF $\beta 2$}

Previous work has shown that transplantation of subcutaneous white adipose tissue (scWAT) from exercise-trained mice into sedentary mice improves metabolism, suggesting that exercise-trained scWAT might produce endocrine factors. A new study in Nature Metabolism identifies transforming growth factor- $\beta 2$ (TGF $\beta 2$ ) as an exercise-induced adipokine that regulates metabolism.

The researchers first performed microarray analysis on scWAT from mice and humans before and after exercise training and identified TGFB2 as an upregulated gene. Furthermore, compared with sedentary participants, exercised-trained humans and mice had significantly higher serum concentrations of TGF $\beta 2$.

In vitro experiments using recombinant TGF $\beta 2$, TGF $\beta 2$ receptor inhibitors and cellular models of WAT, brown adipose tissue (BAT) and muscle suggested that TGF $\beta 2$ stimulates cellular uptake of glucose and fatty acids.

These findings were replicated in vivo, as mice that were fed a normal chow diet and infused with TGF $\beta 2$ using an osmotic pump had improved glucose uptake in mitochondria-enriched oxidative tissues, including muscle, BAT and heart. Moreover, TGF $\beta 2$ infusion improved metabolic parameters and body composition in obese mice that had been fed a high-fat diet (HFD). In addition, TGF $\beta 2$ treatment attenuated adipose tissue inflammation in HFD-fed mice.

Finally, microarray data revealed that lactate metabolic pathways were highly correlated with Tgfb2 expression. Injection of a lactate-lowering agent during exercise training could block the beneficial metabolic effects of TGF $\beta 2$ in mice.

This study has identified a previously undescribed function for TGF $\beta 2$ as an exercise-induced adipokine that regulates glucose and fatty acid metabolism. Moreover, the research has established a new mechanism of lactate-TGF $\beta 2$ signalling by which exercise training can modulate systemic metabolism. TGF $\beta 2$ might represent a new therapeutic target to treat obesity-induced insulin resistance.

Shimona Starling

ORIGINAL ARTICLE Takahashi, H. et al. TGF- $\beta 2$ is an exercise-induced adipokine that regulates glucose and fatty acid metabolism. Nat. Metab. 1, 291-303 (2019) 